\title{
Do patterns of synaptic pruning underlie psychoses, autism and ADHD?
}

\section{Prasanna N. de Silva}

doi: 10.1192/bja.2017.27, Published by Royal College of Psychiatrists, April 2018.

The original publication of this article included an error in the spelling of the title where "psychoses" was spelled as "pychoses". This has been corrected in the original article.

The publisher apologises for this error.

\section{Reference}

de Silva P (2018) Do patterns of synaptic pruning underlie pychoses, autism and ADHD? BJPsych Advances, 24(3): 212-217. doi:10.1192/bja.2017.27. 